PROCEEDINGS OF THE

AMERICAN MATHEMATICAL SOCIETY

Volume 130, Number 9, Pages 2737-2743

S 0002-9939(02)06412-2

Article electronically published on February 4, 2002

\title{
MORSE THEORY, MILNOR FIBERS AND MINIMALITY OF HYPERPLANE ARRANGEMENTS
}

\author{
RICHARD RANDELL
}

(Communicated by Ronald A. Fintushel)

\begin{abstract}
Through the study of Morse theory on the associated Milnor fiber, we show that complex hyperplane arrangement complements are minimal. That is, the complement of any complex hyperplane arrangement has the homotopy type of a CW-complex in which the number of $p$-cells equals the $p$-th betti number. Combining this result with recent work of Papadima and Suciu, one obtains a characterization of when arrangement complements are Eilenberg-Mac Lane spaces.
\end{abstract}

\section{INTRODUCTION}

Let $\mathcal{A}$ be a central arrangement of hyperplanes in $\mathbb{C}^{\ell}$, by which is meant a finite collection $\left\{H_{1}, \ldots, H_{n}\right\}$ where $H_{i}=\alpha_{i}^{-1}(0)$ and each $\alpha_{i}$ is a linear homogeneous form in the variables $\left(z_{1}, \ldots, z_{\ell}\right)$. We call $\mathcal{A}$ an $\ell$-arrangement. We let $M$ be the complement of the union of the hyperplanes

$$
M=\mathbb{C}^{\ell} \backslash \bigcup H_{i}
$$

and we consider the Milnor fibration $Q: M \rightarrow \mathbb{C}^{*}=\mathbb{C} \backslash 0$, with $Q=\prod \alpha_{i}$. This map is actually a fiber bundle with fiber $F=Q^{-1}(1)[1$, called the Milnor fiber. In this paper we will study $F$ via Morse theory, following work of Lê [2]. The Milnor fiber also appears as an $n$-fold cover $p: F \rightarrow M^{*}$ of the complement of the projectivized $M^{*}=M / \mathbb{C}^{*}$, where the action is the restriction of the Hopf action. The Hopf action is free and one sees that the associated bundle is trivial over $M^{*}$ so that $M \cong M^{*} \times \mathbb{C}^{*}$. The Morse functions we consider on $F$ are actually equivariant with respect to the covering transformations of the $n$-fold cover (these covering transformations are generated by the monodromy of the Milnor fibration), thus inducing Morse functions on $M^{*}$, hence a CW-complex structure on the homotopy type of $M^{*}$. Since $M$ has the homotopy type of $M^{*} \times S^{1}$ one gets a $\mathrm{CW}$-complex structure on the homotopy type of $M$ by using the product structure. These CW structures on $M$ and $M^{*}$ have the smallest possible number of cells in each dimension, showing that central and affine arrangement complements are minimal in the sense of Papadima-Suciu [3. From their work a criterion for when the arrangement complement is an Eilenberg-Mac Lane $K(\pi, 1)$-space follows immediately (Corollary 12).

Received by the editors November 29, 2000 and, in revised form, April 16, 2001.

2000 Mathematics Subject Classification. Primary 52C35, 55Q52; Secondary 14N20, 32S22.

Key words and phrases. Hyperplane arrangement, Milnor fiber, Morse theory. 
The number of cells for all these CW-complexes is determined combinatorially by the intersection lattice of the arrangement (see [4] for general background on hyperplane arrangements). However, for arrangements in different path components of the configuration space, one may have varying topology. In our context this would be reflected via the attaching maps in the $\mathrm{CW}$-complexes. In a paper in preparation we study these attaching maps, as well as the behavior of these $\mathrm{CW}$-complexes in lattice isotopic families and in degenerating families.

Papers of Orlik and Terao [5] and Dimca [6] consider Morse theory and the Milnor fiber. In particular, Dimca also shows that arrangement complements are minimal. Our construction follows Lê [2] in using linear sections and readily yields minimality and explicit computation.

I am pleased to thank Alexandru Dimca, Michael Falk and Alexander Suciu for helpful conversations and comments. In particular, A. Dimca pointed out that the equivariant Morse function on $F$ induces a Morse function on $M^{*}$, thereby giving a more straightforward proof of Theorem 3 .

\section{Morse theory on the Milnor FiBer}

We follow Lê 2 throughout this section. By a CW structure on a space $X$, we shall mean a CW-complex homotopy equivalent to $X$. In his work, Lê treats a general hypersurface singularity. For a singularity given by the defining function $Q$ of a hyperplane arrangement the situation is especially pleasant. The basic idea is to obtain a CW structure on the Milnor fiber by taking successive generic linear sections and noting that one goes from a particular section to a section of (complex) dimension one greater by attaching cells of known dimension according to a Morse function, which is simply the distance from a suitably chosen hyperplane section of the Milnor fiber. The result from Lê is

Theorem 1 ([2]). For almost all linear forms $L$, the real-valued function $|L|_{F}$ (which measures the distance from a point to the hyperplane $L=0$ ) is a Morse function (relative to the subset $F \cap\{L=0\}$ ). At each critical point this function has index $\ell-1$.

By a linear change of coordinates we may assume $L=z_{\ell}$, and we will henceforth do so. As in [2], we define the polar curve

$$
\Gamma=\left\{\frac{\partial Q}{\partial z_{1}}=\frac{\partial Q}{\partial z_{2}}=\cdots=\frac{\partial Q}{\partial z_{\ell-1}}=0\right\} \cap\{Q \neq 0\} .
$$

Proposition 2. In the setting of this paper, $\left|z_{\ell}\right|_{F}$ is a relative Morse function provided that the linear subspace $\left\{z_{\ell}=0\right\} \subset \mathbb{C}^{\ell}$ contains no intersection of the hyperplanes of the arrangement other than the origin, and that the set $\Gamma$ is a reduced (possibly empty) curve.

Proof. We check directly that Lê's genericity conditions are satisfied for such $L=z_{\ell}$. First one stratifies $\bigcup H_{i}$ by taking as strata the intersections of the hyperplanes, one stratum for each element of the intersection lattice. This stratification is "bonne" in the sense of [2], and $L$ is transverse to the strata, except $\{0\}$, since $L$ contains no other intersection of hyperplanes. The condition on $\Gamma$ is required for Lê's result to ensure that $\left|z_{\ell}\right|_{F}$ is Morse. See [7] for further discussion.

Such linear forms will be called generic. Note that such a generic form yields an equivariant Morse function (with respect to the covering transformations) on $F$. 
(This group of covering transformations is generated by $\zeta=\exp (2 \pi i / n)$ acting by $\zeta\left(z_{1}, z_{2}, \ldots, z_{\ell}\right)=\left(\zeta z_{1}, \zeta z_{2}, \ldots, \zeta z_{\ell}\right)$.) Therefore, as pointed out by A. Dimca, one obtains a Morse function on the quotient $M^{*}$.

Theorem 3. For $L$ generic, the homotopy type of $F\left(\right.$ resp. $\left.M^{*}\right)$ is obtained by adding a number of $(\ell-1)$-cells to $F \cap\{L=0\}$ (resp. to $M^{*} \cap\{L=0\}$ ). The number of cells added to obtain $F$ is $n$ times the number added to obtain $M^{*}$.

Proof. Clearly the number of critical points of the Morse function $|L|=\left|z_{\ell}\right|$ on $F$ is $n$ times the number of critical points of the induced Morse function on $M^{*}$. The Morse functions on $F$ and $M^{*}$ are not proper, so we must take care. Here we follow the argument given by Lê in [2] for the Milnor fiber $F$. The same argument works for $M^{*}$; one simply projects the sets chosen for $F$ via the map $p: F \rightarrow M^{*}$.

The actual Milnor fiber is $F=Q^{-1}(1)$. We first note that for a sufficiently large $r \gg 0, F$ is diffeomorphic to $F_{r}=F \cap\left\{z_{\ell}<r\right\}$ and homotopy equivalent to $\overline{F_{r}}=F \cap\left\{z_{\ell} \leq r\right\}$. Then for $R$ sufficiently large with respect to $r$, we let $B_{R}$ be the closed ball of radius $R$ in the first $(\ell-1)$-coordinates and note that $F$ has the homotopy type of $F \cap\left(B_{R} \times D_{r}\right)$, where $D_{r}$ is the set of complex numbers $z_{\ell}<r$. Further, from [2, Proposition 2.1], we note that $D_{r}$ and $B_{R}$ can be chosen so that for all $\eta \in D_{r}-\{0\}, F \cap\left\{z_{\ell}=\eta\right\}$ is transverse to $\partial B_{R} \times\{\eta\}$ in $\left\{z_{\ell}=\eta\right\}$.

Then it follows that $\left|z_{\ell}\right|: F \cap\left(B_{R} \times D_{r}\right) \rightarrow D_{r}$ is a proper relative Morse function on a manifold with boundary, and the map on the boundary is a submersion. Therefore $F \cap\left(B_{R} \times D_{r}\right)$ has the homotopy type of $F \cap\left\{z_{\ell}=0\right\}$ with $(\ell-1)$-cells attached. As noted, the same argument works in $M^{*}$ by considering images under the map $p$.

Here is a simple example.

Example 4. Consider the 3-arrangement given by $Q=x(x-y)(x+y-z)$ and consider the form $L=z$. Note that $\{z=0\}$ contains no intersection of the hyperplanes. One computes the polar curve $\Gamma=\{z-2 y=0,3 x-y=0\}$ and thus $|L|_{F}$ is a relative Morse function on $F=Q^{-1}(1)$. The critical points are the solutions of the system of equations

$$
\begin{aligned}
Q & =1, \\
Q_{x} & =0, \\
Q_{y} & =0 .
\end{aligned}
$$

Here as usual, $Q_{x}=\frac{\partial Q}{\partial x}$. One may directly calculate that there are three critical points, each of index two. Thus $F$ is formed by adding three 2-cells to $F \cap\{z=0\}$. The set $F \cap\{z=0\}$ is the Milnor fiber for a 2-arrangement, so we repeat the process, this time taking $L=y$. This time one calculates that there are six critical points of index one, so that $F \cap\{z=0\}$ is formed by adding six 1-cells to $F \cap\{y=z=0\}$. Since this latter set is simply the solutions of $x^{3}=1$, or three points, we obtain a CW structure for the homotopy type of $F$ which has three 0-cells, six 1-cells, and three 2-cells. Similarly, we obtain a CW structure on the homotopy type of $M^{*}$ which has one 0-cell, two 1-cells, and one 2-cell (and of course $M^{*}$ has the homotopy type of the two-torus). Finally, since $M \cong M^{*} \times \mathbb{C}^{*}$ we obtain a $\mathrm{CW}$-complex with the homotopy type of $M$ by using the product structure. The structures thus obtained on $M$ and $M^{*}$ in this case (and always) have the number of cells in each dimension equal to the rank of the homology group in that dimension, and in this 
sense they are minimal structures. A more detailed explicit analysis yields the attaching maps, and one can see that $F$ is a two-torus with a rather inefficient cell structure.

Of course in this and what follows we could phrase results in terms of adding handles, and thus build the diffeomorphism type of the Milnor fiber, not simply the homotopy type.

Next we ask how many critical points (handles, cells added) there are at each stage. Let $b_{p}$ denote the usual betti number.

Theorem 5. The number of cells of dimension $p$ in the $C W$ structure for $M^{*}$ equals $b_{p}\left(M^{*}\right)$. The number of cells of dimension $p$ in the $C W$ structure for $F$ is $n \cdot b_{p}\left(M^{*}\right)$.

Proof. We use induction on $\ell$. For $\ell=1$ the result follows since $F$ consists of $n$ points, $M^{*}$ is a point, and $M \cong M^{*} \times \mathbb{C}^{*}$. For $\ell=2$ we note that $M$ is the complement of a union of complex dimension one subspaces, so that $M$ has the homotopy type of the complement of an $(n, n)$-torus link and $M^{*}$ is an $n$-times punctured two-sphere. Thus $P(M)=(1+t)(1+(n-1) t)$. Observe that $F$ is formed by attaching $c_{1}$ one-cells to $n$ points. To compute $c_{1}$ we note that the EulerPoincaré characteristic $e\left(M^{*}\right)$ is $2-n$ so that $e(F)=n e\left(M^{*}\right)=n(2-n)=n-c_{1}$. Thus $c_{1}=n(n-1)$, which is indeed $n$ times the first betti number of $M^{*}$. The case $\ell=2$ also follows from the general induction step below.

For the general inductive step, we consider the $\ell$-arrangement $\mathcal{A}$ with complement $M$. We choose a generic hyperplane $H$, and consider the arrangement $\mathcal{A}^{H}=\left\{H \cap H_{i}\right\}$ as an arrangement in the $(\ell-1)$-dimensional vector space $H$. Since $H$ is generic, no intersection of hyperplanes from the original arrangement (a flat in the language of arrangement theory) is contained in $H$ (except for $\{0\}$ ). Thus the lattice of $\mathcal{A}^{H}$ is the same as that of the original arrangement $\mathcal{A}$ in ranks (= codimension) 0 through $\ell-2$.

Let $N=H \backslash \bigcup\left(H \cap H_{i}\right)$ and let $N^{*}=N / \mathbb{C}^{*}$. Since the lattices of $\mathcal{A}$ and $\mathcal{A}^{H}$ agree in ranks 0 through $\ell-2, b_{k}(M)=b_{k}(N)$ for all $k=0,1, \ldots, \ell-2$ [4]. Since the Poincaré polynomials satisfy $P\left(M^{*}\right)=P(M) /(1+t)$ and $P\left(N^{*}\right)=P(N) /(1+t)$ it follows that $b_{k}\left(M^{*}\right)=b_{k}\left(N^{*}\right)$ for all $k=0,1, \ldots, \ell-2$. We are attaching $(\ell-1)$ cells to $N^{*}$ to form $M^{*}$. These cells can only change homology groups in degree $(\ell-2)$ or $(\ell-1)$. But since $b_{\ell-2}\left(M^{*}\right)=b_{\ell-2}\left(N^{*}\right)$ all the $(\ell-1)$-cells attached to $N^{*}$ to form $M^{*}$ are added with homologically trivial attaching maps, so that the number of $(\ell-1)$-cells added is $b_{\ell-1}\left(M^{*}\right)$, as desired.

Since $M$ has the homotopy type of $M^{*} \times S^{1}$, we form the product CW structure for the homotopy type of $M$, taking the CW structure on $S^{1}$ consisting of a single 0-cell and a single 1-cell. Since the Poincaré polynomial of $M$ is determined combinatorially by the lattice and $P(M)=P\left(M^{*}\right)(1+t)$, one obtains a combinatorial determination of the number of cells in the CW structure for the homotopy types of $M, M^{*}$, and $F$. We next state a number of corollaries.

Corollary 6. $M$ and $M^{*}$ have the homotopy type of $C W$-complexes which have the minimal number of cells in each dimension. That is, the number of p-cells equals the $p$-th betti number. 
Definition 7 (Papadima-Suciu). A space $X$ is minimal provided that:

i) $X$ is homotopy equivalent to a connected finite-type $\mathrm{CW}$-complex in which the number of $p$-cells equals the $p$-th betti number of $X$.

ii) The homology groups of $X$ are torsion-free.

iii) The cup-product map $\cup: \wedge^{*} H^{1}(X) \rightarrow H^{*}(X)$ is surjective.

Corollary 8. $M$ and $M^{*}$ are minimal.

This result is also obtained by Dimca [6].

Example 9. It is not the case that complements of arbitrary hypersurfaces are minimal. For example, the complement of the plane cusp $\left\{x^{2}=y^{3}\right\}$ deformation retracts to the complement of the trefoil knot in the three-sphere. As observed by [3] any non-trivial knot has a non-minimal complement. To see this, simply note that the first homology group is infinite cyclic (so that $b_{1}=1$ ). If the complement had a $\mathrm{CW}$-complex structure with one cell of dimension one, then the fundamental group would be cyclic. But no non-trivial knot complement has a cyclic fundamental group.

From the above corollary one obtains from [3, Theorem 1.4] the following two results. We let $\pi$ denote the fundamental group of $M$.

Corollary 10. Suppose $Y=K(\pi, 1)$ is a finite-type minimal $C W$-complex, with torsion-free cohomology, generated in degree 1 . Then if $b_{k}(Y)=b_{k}(M)$ for all $k \leq p$,

$$
\pi_{k}(M)=0 \text { for } 1<k<p .
$$

Recall that a (path-connected) space $X$ is a $K(G, 1)$ if and only if its fundamental group is isomorphic to $G$ and its universal cover is contractible. It is natural to make the following

Definition 11. A group $G$ is minimal if and only if there exists a minimal $K(G, 1)$ space.

A well-known problem in the theory of arrangements is to determine under what conditions the complement is a $K(\pi, 1)$ space. A special case of the above corollary is

Corollary 12. The complement $M$ of a complex hyperplane arrangement is a $K(\pi, 1)$ space if and only if $\pi$ is minimal and $b_{k}(K(\pi, 1))=b_{k}(M)$ for all $k$.

Thus whether or not $M$ is a $K(\pi, 1)$ is determined by the group $\pi$ and the lattice. The hypothesis on $\pi$ is necessary, as examples $(Q=x y z(y+z)(x-z)(2 x+y))$ of arrangements are known in which the group homology of the fundamental group is not finitely generated in degree three [9]. It is known [7] Theorem 13] that it is always true that $H^{i}(M)=H^{i}(\pi)$ for $i=1,2$.

Corollary 13. The number of cells in each dimension in this $C W$ structure for $F, M$, and $M^{*}$ is determined by the combinatorics of the arrangement (i.e. the intersection lattice).

Artal Bartolo [8] has found arrangements with lattices yielding the same betti numbers for $M$, but different homology groups in the Milnor fibers. Thus the attaching maps for the cells must differ. 
Corollary 14. Letting $c_{p}$ denote the number of cells in the $C W$ structure as found above for $F$, and setting $C(F)=\sum c_{i}(F) t^{i}$, one has

$$
C(F)=n P\left(M^{*}\right)=n P(M) /(1+t) \text {. }
$$

In Example 4 above, $P(M)=(1+t)^{3}$ and $n=3$.

Finally, we examine more closely the location and number of critical points of the Morse function $|z|$ on $F$. We discuss the situation for $\ell=3$; the general situation is similar. Now the critical points are the solutions of the system

$$
\begin{aligned}
Q & =1, \\
Q_{x} & =0, \\
Q_{y} & =0,
\end{aligned}
$$

in $\mathbb{C}^{3}$ with variables $x, y, z$. We consider the related system of equations

$$
\begin{aligned}
Q & =w^{n}, \\
Q_{x} & =0, \\
Q_{y} & =0,
\end{aligned}
$$

in the complex projective space $\mathbb{C P}^{3}$, with homogeneous coordinates $[x: y: z: w]$. These equations have degree $n, n-1, n-1$ respectively, so that there are $n(n-1)^{2}$ solutions in projective space (counting multiplicities). The critical points are just the solutions with $w \neq 0$.

Now consider a generic arrangement $\mathcal{B}$; that is, no three hyperplanes intersect in a one-dimensional subspace of $\mathbb{C}^{3}$. Then one may easily calculate that the $\mathrm{CW}$ structure on the associated Milnor fiber has $n$ zero-cells, $n(n-1)$ one-cells, and $(n(n-1)(n-2)) / 2$ two-cells. As a check, we calculate the number of solutions of (2) with $w=0$. In this case, $Q=0$, where $Q$ is the defining equation for the arrangement $\mathcal{B}$. Since $Q=\prod \alpha_{i}$, this can only happen when some $\alpha_{i}=0$. Now one easily computes $Q_{x}$ and $Q_{y}$ :

$$
Q_{x}=\sum_{i=1}^{n} \frac{\partial \alpha_{i}}{\partial x} \frac{Q}{\alpha_{i}} \quad \text { and } \quad Q_{y}=\sum_{i=1}^{n} \frac{\partial \alpha_{i}}{\partial y} \frac{Q}{\alpha_{i}} .
$$

Therefore when $\alpha_{i}=0, Q_{x}=0$ if and only if either $a_{j}=0$, for some $j \neq i$, or $\frac{\partial \alpha_{i}}{\partial x}=0$. Similarly, if $\alpha_{i}=0$, then $Q_{y}=0$ if and only if either $a_{j}=0$, for some $j \neq i$, or $\frac{\partial \alpha_{i}}{\partial y}=0$. But if both partials $\frac{\partial \alpha_{i}}{\partial x}$ and $\frac{\partial \alpha_{i}}{\partial y}$ are simultaneously zero, then $\alpha_{i}=z$, which does not hold since we chose the Morse function as $|z|$, where the hyperplane $z=0$ contains no flats of the arrangement. Therefore, solutions of (2) with $w=0$ occur exactly at points (of the $\mathbb{C P}^{2}$ where $w=0$ ) where two $a_{i}$ are zero. Thus, for generic $\mathcal{B}$, there are $\left(\begin{array}{l}n \\ 2\end{array}\right)$ such points, each with multiplicity $n$ (the multiplicity of $Q=0$ ), or $n\left(\begin{array}{l}n \\ 2\end{array}\right)$ solutions counted with multiplicities. Thus there are

$$
n(n-1)^{2}-n\left(\begin{array}{l}
n \\
2
\end{array}\right)=\frac{n(n-1)(n-2)}{2}
$$

solutions of (11), and thus the expected number of critical points. For non-generic 3 -arrangements, there are fewer 2-cells added, hence fewer critical points, hence more solutions of (2) with $w=0$. In the example below we will observe solutions tending to $w=0$ in degenerating families. 
The above argument shows in general (any arrangement, any dimension) that the solutions of the generalization of (2) with $w=0$ occur with two $\alpha_{i}$ equal to zero. Therefore, we have

Proposition 15. The number of critical points, and hence the number of $(\ell-1)$ cells added to form $F$, is given by

$$
n(n-1)^{\ell-1}-\mid\{\text { solutions, with multiplicities, of (2) on }\{w=0\}\} \mid \text {. }
$$

Example 16. Let $Q_{t}=x(x+y)(x-y+t z)$. With $t \neq 0$, the three hyperplanes intersect only at the origin, while for $t=0$, they intersect along the $z$-axis. That is, at the point $[0: 0: 1]$ in homogeneous coordinates for $M^{*} \subset \mathbb{C P}^{2}$. Our earlier example was in fact the case $t=1$; all non-zero values for $t$ exhibit the same behavior. But if $t=0$, the Morse function $|z|$ has no critical points (and the polar curve $\Gamma$ is empty). To see this, note that at a critical point of $|z|$ one must have

$$
\begin{aligned}
Q & =x(x+y)(x-y)=x^{3}-x y^{2}=1, \\
Q_{x} & =(x+y)(x-y)+x(x-y)+x(x+y)=3 x^{2}-y^{2}=0, \\
Q_{y} & =x(x-y)-x(x+y)=-2 x y=0 .
\end{aligned}
$$

The third equation shows that $x=0$ or $y=0$. But $x=0$ is clearly impossible, from the first equation, while $y=0$ forces $x=0$ in the second equation, again impossible by the first equation. So in fact $F_{0}$ has the homotopy type of a 1-complex, obtained by removing (or not adding) the three two-cells required to form $F_{1}$.

\section{REFERENCES}

[1] Milnor, J., Singular points of complex hypersurfaces, Annals of Math. Studies 61, Princeton University Press, 1968. MR 39:969

[2] Lê, D. T., Calcul du nombre de cycles évanouissants d'une hypersurface complexe, Ann. Inst. Fourier, Grenoble 23, 4 (1973), 261-270. MR 48:8838

[3] Papadima, S. and Suciu, A., Higher homotopy groups of complements of complex hyperplane arrangements, preprint, arXiv:math.AT/0002251

[4] Orlik, P., and Terao, H., Arrangements of Hyperplanes, Grundlehren der mathematischen Wissenschaften 300, Springer Verlag, 1992. MR 94e:52014

[5] _ Arrangements and Milnor fibers, Math. Ann. 301, (1995), 211-235. MR 96f:52014

[6] Dimca, A., Hypersurface complements, Milnor fibers and minimality of arrangements, preprint, arXiv:math.AG/0011222.

[7] Randell, R., Homotopy and group cohomology of arrangements, Topology and its Applications 78, (1997), 201-213. MR 98f:52014

[8] Artal Bartolo, E., Combinatorics and topology of line arrangements in the complex projective plane, Proc. Amer. Math. Soc., 121, (1994), 385-390. MR 94h:14020

[9] Arvola, B., Arrangements and cohomology of groups, preprint, 1992.

Department of Mathematics, University of Iowa, Iowa City, Iowa 52242

E-mail address: randell@math.uiowa.edu 